\title{
A Statistical Model for Predicting the Liquid Steel Temperature in Ladle and Tundish by Bootstrap Filter
}

\author{
Sho SONODA, ${ }^{1)}$ Noboru MURATA, ${ }^{1)}$ Hideitsu HINO, ${ }^{1)}$ Hiroshi KITADA ${ }^{2)}$ and Manabu KANO ${ }^{3)}$ \\ 1)Waseda University, 3-4-1 Okubo, Shinjuku-ku, Tokyo, 169-8555 Japan. Email: s.sonoda0110@toki.waseda.jp,noboru.murata@ \\ eb.waseda.ac.jp, hideitsu.hino@toki.waseda.jp $\quad$ 2) Sumitomo Metal Industries, Ltd., 1-8 Fusocho, Amagasaki-shi, Hyogo, \\ 660-0891 Japan.Email: kitada-hrs@sumitomometals.co.jp \\ 3) Kyoto University, Yoshida-Honmachi, Sakyo-ku, Kyoto, 606-8501 Japan. Email: manabu@i.kyoto-u.ac.jp
}

(Received on January 4, 2012; accepted on February 1, 2012)

\begin{abstract}
A statistical model for predicting the liquid steel temperature in the ladle and in the tundish is developed. Given a large data set in a steelmaking process, the proposed model predicts the temperature in a seconds with a good accuracy. The data are divided into four phases at the mediation of five temperature measurements: before tapping from the converter (CV), after throwing ferroalloys into the ladle, before and after the Ruhrstahl-Heraeus $(\mathrm{RH})$ processing, and after casting into the tundish in the continuous casting (CC) machine. Based on the general state space modeling, the bootstrap filter predicts the temperature phase by phase. The particle approximation technique enables to compute general-shaped probability distributions. The proposed model gives a prediction not as a point but as a probability distribution, or a predictive distribution. It evaluates both uncertainty of the prediction and ununiformity of the temperature. It is applicable to sensitivity analysis, process scheduling and temperature control.
\end{abstract}

KEY WORDS: statistical modeling and simulation; liquid steel temperature control; general state space model; bootstrap filter; steelmaking.

\section{Introduction}

In continuous casting, the liquid steel temperature in the ladle and in the tundish is crucial for achieving high product quality. When the temperature is too high, an injurious influence on the inner structure of the steel occurs and it can cause defects in the slabs. On the other hand, when the temperature is too low, clogging of nozzles can occur. Since the last operation point for controlling the temperature through the process is apart from the tundish, it is important to analyze which operation affects the temperature.

Past attempts to predict the temperature are divided into two approaches: physical and statistical. The former, mainly based on the thermodynamics, has long history and there are many studies. ${ }^{1-20)}$ However, physical models require complex numerical techniques like finite difference methods $(\mathrm{FDM})^{1-12)}$ and finite element methods (FEM). ${ }^{13,21)}$ Moreover, physical calculation of liquid steel temperature requires the simulation of thermo-fluid behaviors like vortex and convection, which is time-consuming.

On the other hand, a purely statistical model has been developed for predicting the liquid steel temperature in the ladle furnace. ${ }^{22)}$ It uses a variant of neural network called the extreme learning machine (ELM) and predicts the temperature with the power consumption, the initial temperature, the ladle state, etc. In general, predictions by statistical models are faster than those by physical ones based on thermo-fluid dynamics.

Attempting to reduce the amount of computation, $\mathrm{N}$.
Gupta and S. Chandra ${ }^{23)}$ have introduced a statistical model as a part of a physical one. Meanwhile T. Okura et al. ${ }^{24)}$ has reported that a multiple regression model has achieved a competitive accuracy to a physical ladle model.

Those attempts, though, make only point estimation of the temperature. On the contrary, our model aims to predict the temperature as a probability distribution of predictive values, termed a predictive distribution. It provides not only predictions but also degree of its deviation, or variance. This advantage is applicable to sensitivity analysis, process scheduling and temperature control.

The rest of this paper is organized as follows. In Sec. 2, a statistical prediction model based on the bootstrap filter is established for predicting the liquid steel temperature by splitting the prediction procedure into four phases. In Sec. 3 , preliminary procedures estimating deterministic transition functions and variance parameters are presented. In Sec. 4, the proposed model is evaluated by the data set which is obtained from the operations of actual steelmaking plants. Finally, the last section concludes this paper.

\section{Statistical Modeling and Prediction Method}

\subsection{Data Overview and Four Phases of Process}

The data set has 2840 lines. A line of data is associated with one of 18 ladles carried from the converter (CV) to the continuous casting (CC) machine. It has 78 items, such as liquid steel temperatures, concentrations (conc.) of the components in steel, elapsed times of every process, solvent 
quantities and ladle states. It is known that estimation results of parameters of a prediction model vary according to ladle. In order to estimate the prediction model which has 78 regressors and varies according to at most 18 different ladles, 2840 lines are not enough. Therefore we split the prediction problem into small ones. As shown in Fig. 1, items and their corresponding processes are classified into four phases. They are separated at five temperature measurements: before tapping from $\mathrm{CV}$, after throwing ferroalloys into the ladle, before and after the Ruhrstahl-Heraeus (RH) processing, and after casting into the tundish in the $\mathrm{CC}$ machine. These temperature measurements are numbered as $t=0, \cdots, 4$ and a process lying between $t-1$ and $t$ is called phase $t$. The observed temperature at measurement $t$ is denoted by $X_{t}$. Other items classified in phase $t$ are collectively denoted by $Y_{t}$. As discussed later, these uppercase variables are regarded as random variables. While lowercase notations, for example $x_{t}$ and $y_{t}$, are regarded as realized values of corresponding random variables written in uppercase. Therefore, a line of data is written as $\left(x_{0: 4}, y_{1: 4}\right)$, where $x_{s: t}$ and $y_{s: t}$ are abbreviations of $\left\{x_{s}, \cdots, x_{t}\right\}$ and $\left\{y_{s}, \cdots, y_{t}\right\}$ respectively. At last, the data set is written as $\left\{\left(x_{0: 4}^{(m)}, y_{1: 4}^{(m)}\right)\right\}_{m=1}^{M}$ where $m$ is the index of lines.

\subsection{General State Space Model}

\subsubsection{Overview}

The general state space model consists of Markovian internal state variables $T_{t}(t=0,1, \cdots)$ and their corresponding observation variables $X_{t}(t=0,1, \cdots)$. Here $T_{t-1}$ transits to $T_{t}$ according to a state transition model, or a conditional probability distribution:

$$
T_{t}-P\left(T_{t} \mid T_{t-1}\right),
$$

and $X_{t}$ is generated from $T_{t}$ according to an observation model, or a conditional probability distribution:

$$
X_{t}-P\left(X_{t} \mid T_{t}\right)
$$

In this paper, $X_{t}$ is an observed liquid steel temperature, and $T_{t}$ is an unobserved real liquid steel temperature. Although both represent liquid steel temperature in the ladle and tundish, they are distinguished from each other, because $X_{t}$ is observed with noise, while $T_{t}$ is not observed. We regard a temperature value appeared in the data as a realization of $X_{t}$, that is, $x_{t}$. Whereas $T_{t}$ has no $t_{t}$ 's and therefore it needs to be estimated from $X_{t}$.

Based on the general state space model, the bootstrap

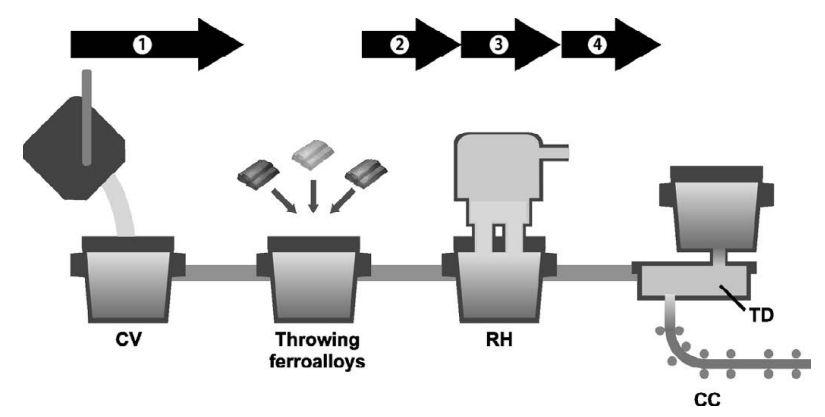

Fig. 1. Process Flows Split into Four Phases. filter, ${ }^{25)}$ a kind of sequential Monte Carlo method, is used to estimate $T_{t}$ from $X_{t}$. In particular, when the state transition model is linear and the observation model is Gaussian, whole probability distributions are explicitly computable and the estimation method comes down to the Kalman filter. ${ }^{26)}$ On the contrary, since distributions are not always Gaussian and transition models are not always linear, we employ the bootstrap filter, which can handle generalshaped distributions and non-linear transitions.

\subsubsection{State Transition Model}

We model the transition from $T_{t-1}$ to $T_{t}$ by the composition of a deterministic transition process and a stochastic fluctuation. Given $T_{t-1}$ and other associated explanatory variables $Y_{t}, T_{t-1}$ first transits to $f_{t}\left(T_{t-1}, Y_{t}\right)$ with a deterministic transition function $f_{t}$. Some statistical regression models can be candidates for representing $f_{t}$. Then, a system noise $\varepsilon_{t}$ is added to $f_{t}\left(T_{t-1}, Y_{t}\right)$ to express the residual which $f_{t}$ can not predict. Hence, the state transition is formulated as

$$
T_{t}=f_{t}\left(T_{t-1}, Y_{t}\right)+\varepsilon_{t}
$$

Assuming that $\varepsilon_{t}$ is a Gaussian with mean 0 and variance $\sigma_{t}^{2}$, the state transition model is written as

$$
P\left(T_{t} \mid T_{t-1}, Y_{t}\right)=N\left(T_{t} ; f_{t}\left(T_{t-1}, Y_{t}\right), \sigma_{t}^{2}\right),
$$

where $\mathrm{N}\left(\cdot ; \mu, \sigma^{2}\right)$ denotes a Gaussian with mean $\mu$ and variance $\sigma^{2}$. The method for estimating the variance parameter $\sigma_{t}^{2}$ is described in Sec. 3.

\subsubsection{Observation Model}

We model the observation of $X_{t}$ from $P\left(T_{t}\right)$ in a two-step way. First, we select a single point of steel to measure. This selection is associated with a sampling from $P\left(T_{t}\right)$. Second, we measure its temperature with an observation error $\xi_{t}$. Therefore, their relation is formulated as

$$
X_{t}=T_{t}+\xi_{t}
$$

Assuming that $\xi_{t}$ is also a Gaussian with mean 0 and variance $\tau_{t}^{2}$, the observation model is written as

$$
P\left(X_{t} \mid T_{t}\right)=N\left(X_{t} ; T_{t}, \tau_{t}^{2}\right) .
$$

The method for estimating the variance parameter $\tau_{t}^{2}$ is described in Sec. 3.

\subsection{Predictive Distribution}

The predictive distribution gives a prediction not as a point but as a probability distribution. In this study, the prediction of $T$ is given as a predictive distribution $P(T)$. We can see $P(T)$ from two different viewpoints: statistical and physical ones. From the statistical viewpoint, $P(T)$ simply represents uncertainty of the prediction. Namely, a large variance of $P(T)$ indicates that the prediction is inaccurate. On the other hand, from the physical viewpoint, $P(T)$ roughly represents possible ununiformity of the liquid steel temperature in the ladle and tundish. That is, a large variance of $P(T)$ suggests that there would be both a cool part and a hot part. In particular, from either viewpoint, a small variance implies the liquid steel is in a better condition.

\subsection{Sequential Prediction by Bootstrap Filter}

Figure 2 shows the graphical model ${ }^{27)}$ which depicts the 


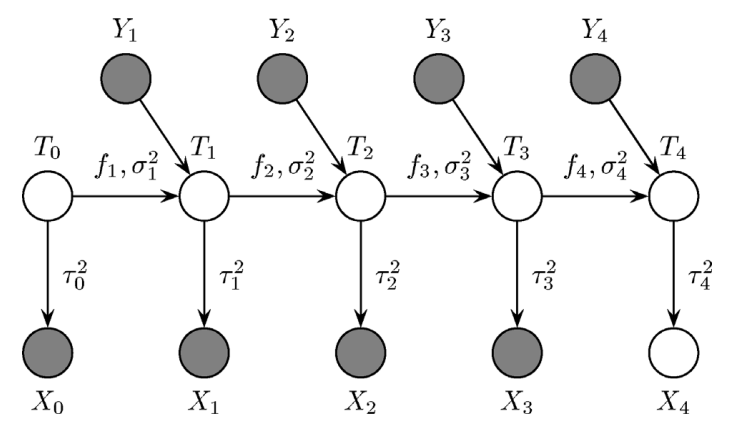

Fig. 2. Graphical Model.

relations of random variables appeared in this paper. Filled and open nodes indicate that corresponding random variables are observed and not observed respectively.

Our goal is to compute the probability distribution $P$ $\left(T_{4} \mid X_{0: 3}, Y_{1: 4}\right)$. Given the Markovian assumption that $T_{t}$ depends only on $T_{t-1}$ :

$$
P\left(T_{t} \mid T_{0: t-1}\right)=P\left(T_{t} \mid T_{t-1}\right), \ldots
$$

starting from $P\left(T_{0}\right)$, four iterations of computation from $P$ ( $\left.T_{t-1}\right)$ to $P\left(T_{t}\right)$ lead to $P\left(T_{4}\right)$. The bootstrap filter repeats the following two steps alternately. The first step is modification. Given a prior distribution $P\left(T_{t-1}\right)$ and an observation variable $X_{t-1}, P\left(T_{t-1}\right)$ is modified to the posterior distribution $P\left(T_{t-1} \mid X_{t-1}\right)$ according to the observation model Eq. (6). It is calculated by Bayes' formula:

$$
P\left(T_{t-1} \mid X_{t-1}\right) \propto P\left(X_{t-1} \mid T_{t-1}\right) P\left(T_{t-1}\right) .
$$

The second step is transition. Given the posterior distribution $P\left(T_{t-1} \mid X_{t-1}\right)$, a predictive distribution $P\left(T_{t}\right)$ is calculated according to the transition model given by Eq. (4).

As iterations proceed, initial information decreases and the estimation accuracy increases phase by phase. Therefore the initial setting $P\left(T_{0}\right)$ is not much sensitive to the prediction. In our experiments, we employ the uniform distribution on the interval which covers whole observed temperature $x_{0}$ 's.

\subsection{Particle Approximation of Probability Distribution}

The particle approximation represents a general-shaped probability distribution by the density of many particles. That is, $P\left(T_{t}\right)$ is approximated by a collection of $N$ particles $\left\{T_{t}^{(n)}\right\}_{n=1}^{N}$ where each particle $T_{t}^{(n)}$ is sampled from $P\left(T_{t}\right)$. To simplify the notations, we identify a collection of particles with its representing distribution and call it simply a distribution.

By means of the particle approximation, Bayes' formula is calculated easily. Given a prior distribution $\left\{T_{t}^{(n)}\right\}_{n=1}^{N} \sim$ $P\left(T_{t}\right)$ and an observation variable $X_{t}$, we compute a conditional probability value $W_{t}^{(n)}:=P\left(X_{t} \mid T_{t}^{(n)}\right)$ which represents the occurrence probability of $X_{t}$ given $T_{t}^{(n)}$. Then we resample $\left\{T_{t}^{\prime^{(n)}}\right\}_{n=1}^{N}$ from $\left\{T_{t}^{(n)}\right\}_{n=1}^{N}$ with replacement in such a way that the frequency of $T_{t}^{(n)}$ is proportional to $W_{t}^{(n)}$. Thereby $\left\{T_{t}^{\prime(n)}\right\}_{n=1}^{N}$ represents the posterior distribution $P\left(T_{t} \mid X_{t}\right)$.
Algorithm 1. Prediction Algorithm by Bootstrap Filter.

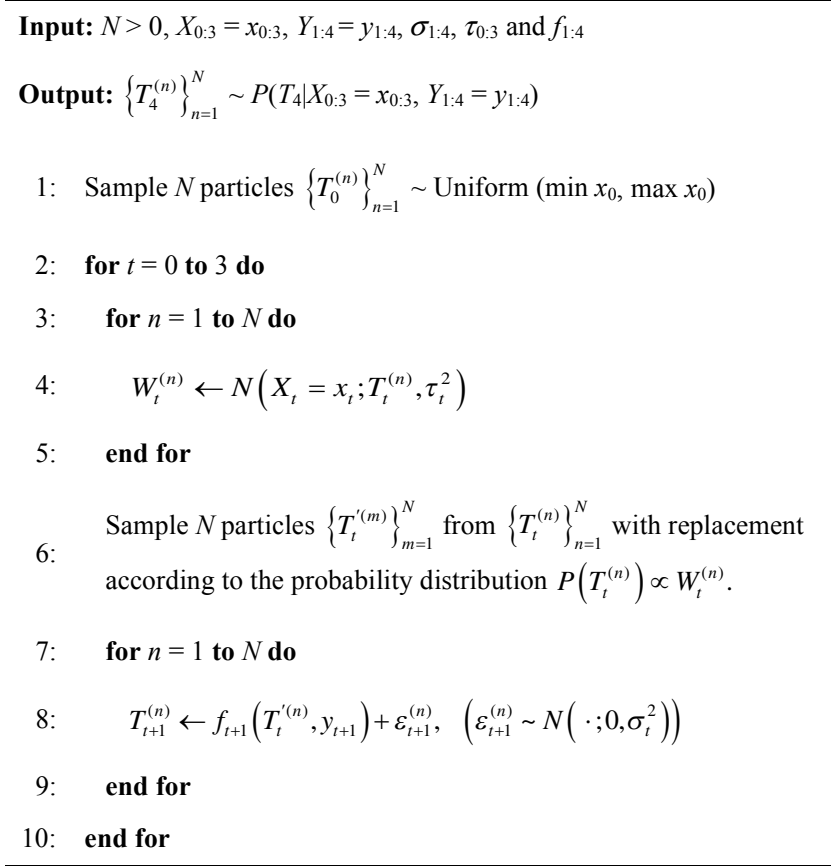

It is also easy to compute transition steps with particles. Given the posterior distribution $\left\{T_{t}^{\prime(n)}\right\}_{n=1}^{N} \sim P\left(T_{t} \mid X_{t}\right)$, just a pointwise application of Eq. (3)

$$
T_{t+1}^{(n)}=f_{t+1}\left(T_{t}^{\prime(n)}, Y_{t+1}\right)+\varepsilon_{t+1}^{(n)}, \quad\left(\varepsilon_{t+1}^{(n)} \sim N\left(\cdot ; 0, \sigma_{t+1}^{2}\right)\right) \ldots
$$

generates the next probability distribution $\left\{T_{t+1}^{(n)}\right\}_{n=1}^{N} \sim P\left(T_{t+1}\right)$.

In addition, the expectation value of a function $g\left(T_{t}\right)$ with respect to $P\left(T_{t}\right)$ is given by the Monte Carlo integration:

$$
\begin{aligned}
\mathbb{E}\left[g\left(T_{t}\right)\right] & =\int g\left(T_{t}\right) P\left(T_{t}\right) d T_{t} \\
& \approx \frac{1}{N} \sum_{n=1}^{N} g\left(T_{t}^{(n)}\right), \quad\left(\left\{T_{t}^{(n)}\right\}_{n=1}^{N} \sim P\left(T_{t}\right)\right) .
\end{aligned}
$$

To sum up theories and techniques described so far, the prediction process for one ladle is summarized in Algorithm 1 . Note that the deterministic transition function $f_{t}$ and variance parameters $\sigma_{t}^{2}, \tau_{t}^{2}$ have to be learned from data beforehand. Their estimation methods will be mentioned in Sec. 3 .

\section{Preliminary Estimations}

\subsection{Deterministic Transition Function $\boldsymbol{f}_{\boldsymbol{t}}$}

Since $T_{t-1}$ and $T_{t}$ are not observed, $f_{t}$ of $T_{t-1}$ is estimated using $x_{t-1}$ 's and $x_{t}$ 's. That is, we train $f_{t}$ as to minimize the square error

$$
\sum_{m=1}^{M}\left|x_{t}^{(m)}-f_{t}\left(x_{t-1}^{(m)}, y_{t}\right)\right|^{2},
$$

instead of using $t_{t-1}$ 's and $t_{t}$ 's. We regard the estimation result as a substitute for true $f_{t}$. To be exact, the estimation is performed with not only the system noise $\varepsilon_{t}$ but also observation errors $\xi_{t-1}$ and $\xi_{t}$. Our substitution corresponds to ignore the effects of observation errors. We expect that $\varepsilon_{t}$ absorbs inconsistencies caused by the ignorance. The experimental results support our expectation. 
Note that by means of physical modeling, $f_{t}$ can be directly modeled as a function of $T_{t-1}$, and the substitution problem would not happen in that case. However, since physical modeling and its computation are usually diffcult, we deal with only statistical models.

\subsection{Variance Parameters $\sigma_{t}^{2}$ and $\tau_{t}^{2}$}

Variance parameters $\sigma_{t}^{2}$ and $\tau_{t}^{2}$ can be determined in many ways. Here we explain two methods: the maximum likelihood estimation (MLE) and the just-in-time method (JIT). In the experiments, we employ MLE.

\subsubsection{Maximum Likelihood Estimation (MLE)}

In MLE, parameters $\sigma_{t}^{2}$ and $\tau_{t}^{2}$ are determined by maximizing the likelihood of training data $\left\{\left(x_{0: 4}^{(m)}, y_{1: 4}^{(m)}\right)\right\}_{m=1}^{M}$. The likelihood function is given by

$$
L\left(\sigma_{1: 4}^{2}, \tau_{0: 4}^{2}\right)=\prod_{m=1}^{M} P\left(x_{0: 4}^{(m)} \mid y_{1: 4}^{(m)} ; \sigma_{1: 4}^{2}, \tau_{0: 4}^{2}\right),
$$

where

$$
\begin{aligned}
P\left(X_{0: 4} \mid Y_{1: 4} ; \sigma_{1: 4}^{2}, \tau_{0: 4}^{2}\right) & =P\left(X_{0}\right) \prod_{t=1}^{4} P\left(X_{t} \mid X_{0: t-1}, Y_{1: t} ; \sigma_{1: t}^{2}, \tau_{0: t}^{2}\right) \\
& =P\left(X_{0}\right) \prod_{t=1}^{4} P\left(X_{t} \mid X_{t-1}, Y_{t} ; \sigma_{t}^{2}, \tau_{t-1: t}^{2}\right)
\end{aligned}
$$

and

$$
\begin{aligned}
& P\left(X_{t} \mid X_{t-1}, Y_{t} ; \sigma_{t}^{2}, \tau_{t-1: t}^{2}\right) \\
& \quad=\int P\left(X_{t} \mid T_{t} ; \tau_{t}^{2}\right) P\left(T_{t} \mid T_{t-1}, Y_{t} ; \sigma_{t}^{2}\right) P\left(T_{t-1} \mid X_{t-1} ; \tau_{t-1}^{2}\right) d T_{t} d T_{t-1} .
\end{aligned}
$$

The last integration is calculated by particle approximation as

$$
\begin{aligned}
P\left(X_{t} \mid X_{t-1}, Y_{t}\right) & \approx \frac{1}{N} \sum_{n=1}^{N} \int P\left(X_{t} \mid T_{t}\right) P\left(T_{t} \mid T_{t-1}^{(n)}, Y_{t}\right) d T_{t} \\
& \approx \frac{1}{N^{2}} \sum_{n=1}^{N} \sum_{m=1}^{N} P\left(X_{t} \mid T_{t}^{(m, n)}\right),
\end{aligned}
$$

where $\left\{T_{t-1}^{(n)}\right\}_{n=1}^{N} \sim P\left(T_{t-1}\right)$ and $\left\{T_{t}^{(m, n)}\right\}_{m=1}^{N} \sim P\left(T_{t} \mid T_{t-1}^{(n)}, Y_{t}\right)$.

Maximization of the likelihood function $\mathrm{L}$ of $\sigma_{\mathrm{t}}^{2}$ and $\tau_{\mathrm{t}}^{2}$ is achieved by simultaneous perturbation stochastic approximation (SPSA). ${ }^{28)}$

\subsubsection{Just-In-Time Method (JIT)}

While we assume that the variance $\tau_{\mathrm{t}}^{2}$ of the observation error does not depend on data line, the variance $\sigma_{\mathrm{t}}^{2}$ of the system noise differ in each line. Once $\tau_{0: 4}^{2}$ are estimated by MLE, the JIT method is a simple and quick way to obtain $\sigma_{1: 4}^{2}$. First, given a target line $\left(x_{t-1}^{(0)}, y_{t}^{(0)}\right)$, we collect $k$ nearest training data lines $\left\{\left(x_{t-1}^{(j)}, y_{t}^{(j)}, x_{t}^{(j)}\right)\right\}_{j=1}^{k}$ to the target. Second, we compute the squared mean prediction error

$$
\frac{1}{k} \sum_{j=1}^{k}\left|y_{t}^{(j)}-f_{t}\left(x_{t-1}^{(j)}, y_{t}^{(j)}\right)\right|^{2}
$$

as an estimator $\widehat{\sigma_{t}^{2}}$. Even though this method must execute the procedure for every new data line, it is even faster than MLE. Meanwhile we must select carefully the number of neighbors and the distance among data lines.

\section{Experimental Results}

\subsection{Data Description}

The data set was obtained from the operations of actual steelmaking plants. The values of time, weight, temperature and other physical properties in the data were scaled for the sake of confidentiality of the original data. Randomly chosen $80 \%(\approx 2200)$ of data are used to train $f_{t}$ and estimate $\sigma_{t}^{2}$, $\tau_{t}^{2}$. The rest $20 \%(\approx 560)$ are used to test the prediction accuracy.

\subsection{Settings and Preliminary Experiments}

\subsubsection{Selection of Deterministic Transition Function}

Table 1 compares the pointwise prediction accuracies of five statistical regression models: linear regression, three layered perceptron, ${ }^{29)}$ projection pursuit regression (PPR), ${ }^{30}$ recursive partitioning and regression trees (RPART), ${ }^{31)}$ and Random Forest. ${ }^{32)}$ Random Forest is an ensemble learning method with regression trees which is able to select explanatory variables automatically. In this comparison, $80 \%$ of training data, namely $64 \%$ of whole data, are used to train $f_{t}$ and the rest $20 \%$ are used to evaluate their generalization errors

$$
\left(\frac{1}{M} \sum_{m=1}^{M}\left|x_{t}^{(m)}-f_{t}\left(x_{t-1}^{(m)}, y_{t}^{(m)}\right)\right|^{2}\right)^{\frac{1}{2}}, \quad(t=1, \cdots, 4) .
$$

Three layered perceptrons have hidden units ranging from 500 to 1000 depending on phases. Random Forests have 300 regression trees in each phase and the maximum size of terminal nodes, i.e. the number of observations represented by the terminal nodes, is restricted to five. The result shows that Random Forest score the least generalization error at every phase. Therefore we choose Random Forest for $f_{t}$ in every phase.

\subsubsection{Selection of Explanatory Variables}

Table 2 shows explanatory variables on which Random Forests scored high important weights. According to the results, we select about 10 variables as $Y_{t}$ in every phase. Quantity of the components in steel is important in all phases. It is reasonable because the components determine the specific heat of steel. On the other hand, solvent influence is relatively unimportant. At phases 1 to 3, ladle state variables, such as the number of use and waiting time, are selected. They are related to ladle's heat-retention property.

Table 1. Generalization Errors for Test Data.

\begin{tabular}{ccccc}
\hline Model & Phase 1 & Phase 2 & Phase 3 & Phase 4 \\
\hline Linear Regression & 7.56 & 4.23 & 3.27 & 2.64 \\
Three Layered Perceptron & 10.2 & 8.46 & 5.92 & 4.98 \\
Projection Pursuit Regression & 7.33 & 3.59 & 3.49 & 2.45 \\
RPART & 7.39 & 4.39 & 3.75 & 2.91 \\
Random Forest & $\mathbf{6 . 3 4}$ & $\mathbf{3 . 1 0}$ & $\mathbf{3 . 2 5}$ & $\mathbf{2 . 3 8}$ \\
\hline
\end{tabular}




\subsubsection{Estimation of Variance Parameters}

Table 3 shows estimation results of the parameters by MLE. As phases proceed, $\sigma_{\mathrm{t}}^{2}$ gets small. It suggests that the prediction accuracy of $f_{t}$ increases. On the other hand, $\tau_{\mathrm{t}}^{2}$ is nearly constant with respect to $t$. It implies that the observation error does not depend on measurement time.

\subsubsection{Setting the Number of Particles}

In our experiments, the number $N$ of particles $\left\{T_{t}^{(n)}\right\}_{n=1}^{N}$ is set to 1000 in every phase $(t=1, \cdots, 4)$. The computation time with 1000 particles is fast enough for on-the-spot use. Density plots of 1000 particles, for example shown in the main experiments (Fig. 3), demonstrate that distributions are well approximated.

\subsubsection{Representing Particle Approximations}

Although the proposed model gives predictive distributions, single point selection from the distribution is needed to compare it with other pointwise models. We employ the expectation value of the distribution

$$
\begin{aligned}
\mathbb{E}\left[T_{4} \mid X_{0: 3}, Y_{1: 4}\right]=\int T_{4} P\left(T_{4} \mid X_{0: 3}, Y_{1: 4}\right) d T_{4} \\
\approx \frac{1}{N} \sum_{n=1}^{N} T_{4}^{(n)}, \quad\left(T_{4}^{(n)} \sim P\left(T_{4}=s \mid X_{0: 3}, Y_{1: 4}\right)\right),
\end{aligned}
$$

Table 2. Explanatory Variables Selected by Random Forest.

\begin{tabular}{cl}
\hline$t$ & \multicolumn{1}{c}{$Y_{t}$} \\
\hline 1 & $\begin{array}{l}\text { Ladle states, Conc. of the components in steel before tapping, } \\
\text { Weight of the ferroalloys thrown in the ladle, etc. }\end{array}$ \\
2 & $\begin{array}{l}\text { Ladle states, Conc. of the components in steel after throwing ferroal- } \\
\text { loys, transferring time from CV to RH, etc. }\end{array}$ \\
3 & $\begin{array}{l}\text { Ladle states, Target conc. of the components in steel after RH, Conc. } \\
\text { of the components in steel after throwing ferroalloys, etc. }\end{array}$ \\
4 & $\begin{array}{l}\text { Transferring time from RH to CC, Elapsed time after casting, Casting } \\
\text { time, Conc. of the components in steel after throwing ferroalloys, etc. }\end{array}$
\end{tabular}

Table 3. Optimized Parameters Estimated by MLE

\begin{tabular}{ccccc}
\hline & $t=1$ & $t=2$ & $t=3$ & $t=4$ \\
\hline$\sigma_{t}$ & 5.65 & 2.38 & 3.72 & 1.68 \\
$\tau_{t}$ & 1.78 & 1.78 & 1.42 & 1.80 \\
\hline
\end{tabular}
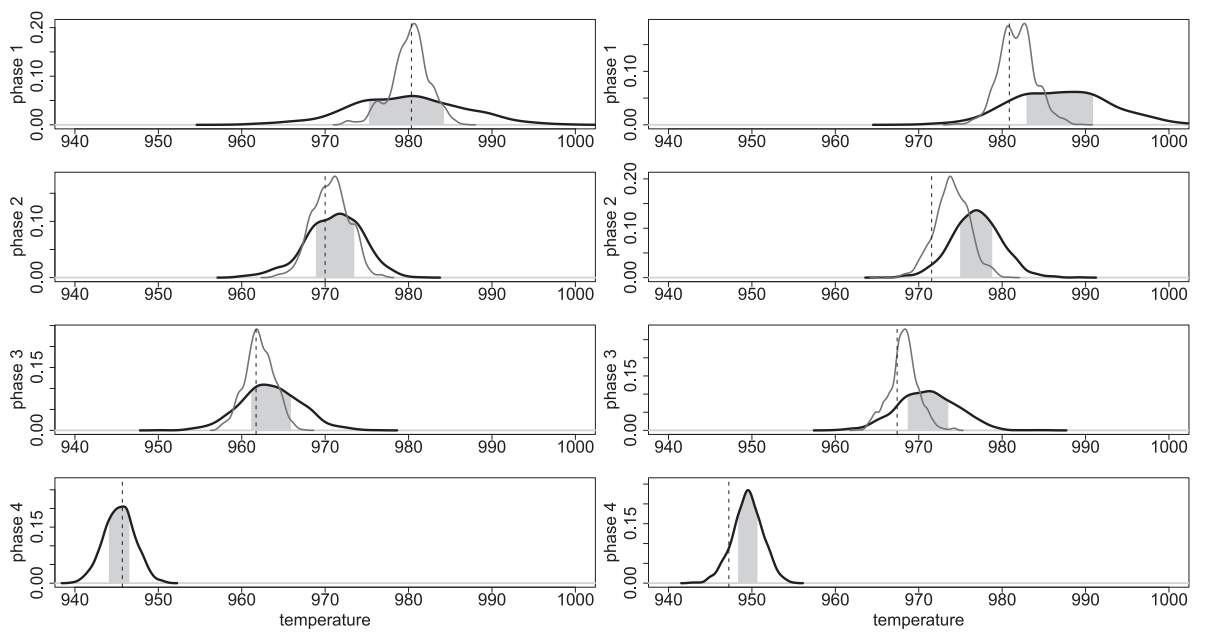

Fig. 3. Examples of Sequential Estimation by Bootstrap Filter.

as a representative value, which is abbreviated to $\mathbb{E}\left[T_{4}\right]$. Note that the expectation is appropriate for a representative when the distribution is unimodal. Distributions appeared in our experiments are observed to be unimodal, therefore our choice is reasonable.

\subsection{Main Results}

Two examples of estimation results are shown in Fig. 3. The thick line shows the prior distribution $P\left(T_{t}\right)$ and the thin line shows the posterior distribution $P\left(T_{t} \mid X_{t}\right)$. The vertical dashed line indicates the observed temperature $x_{t}$. As phases proceed, distributions shift from right to left, that means the temperature gets lower gradually. It is also observed that the variance gets smaller, that implies the prediction accuracy increases phase by phase. In the left hand side case, the expectation value $\mathbb{E}\left[T_{4}\right]$ of the predictive distribution is 945.3 and the actual observed temperature $x_{4}$ is 945.7 , which is inside the $50 \%$-percentile of the predictive distribution. It suggests that the prediction is success. On the other hand, in the right hand side case, $\mathbb{E}\left[T_{4}\right]=949.4$ while $x_{4}=947.2$. In this case, the modification step did not work well at every phase and failed to predict the temperature at phase 4. As for whole test data (147 data lines omitting NA), the root mean squared prediction error is 2.5 . It is comparable accuracy to the best point estimation model shown in Table 1 at phase 4 . Note that our model gives not only an accurate prediction but also its variance, or a kind of confidence interval.

\section{Discussions and Concluding Remarks}

A statistical model for predicting the liquid steel temperature by bootstrap filter has been proposed. In order to predict the temperature effciently from as many as 78 items, the prediction process has been split into four phases. Based on the general state space modeling, the prediction has been made by bootstrap filter with predictive distributions. The proposed model has performed in a comparable accuracy to the best pointwise prediction model. Although our model requires more computations than point estimation methods, total computation time has been still so fast that on-the-spot use is possible.

Higher accuracy of the prediction would be achieved by better selection of deterministic transition functions. In fact, 
the proposed model is capable of employing not only statistical models but also physical ones, therefore further investigations on latters would be promising.

Meanwhile the proposed model excels at predicting the temperature with predictive distributions. From the predictive distribution, we can evaluate the worst case of temperature ununiformity. It would be applicable to sensitivity analysis, process scheduling and liquid steel temperature control. For example of sensitivity analysis, if we perturb explanatory variables during a prediction process one by one, prediction results may vary and we can detect sensitive variables which amplify the temperature variance. Our preliminary experiment for sensitivity analysis has shown that the amount of oxygen used to heat up steel after RH tends to increase the variance of predictive distributions.

Other examples of applications are process scheduling and temperature control. Since the proposed model computes in a short seconds, it is possible to make predictions on-line at the steelmaking plant. If operators simulate time delaying of a process and evaluate the worst acceptable enhancement of temperature ununiformity, they could know the deadline of the process. This is applicable to process scheduling. Besides, if operators repeat random perturbing simulations, it would be possible to find a better recipe of operations which achieves preferably uniform temperature. This is applicable to temperature control. The applications mentioned above remain to be our important future works.

\section{Acknowledgement}

This study has been partially supported by the grant from ISIJ as an activity of research group, "High Precision Process Control via Large Scale Database and Simulation Models". The authors are grateful to the editor and reviewers for valuable comments.

\section{REFERENCES}

1) T. Fredman: Scand. J. Metall., 29 (2000), 232.
2) R. Alberny and A. Leclercq: Proc. of Conf. on Mathematical Process Models in Iron and Steelmaking, Metal Soc., London, (1973), 151.

3) M. Omotani, L. Heaslip and A. Maclean: Iron Steelmaker, 10 (1983), 29.

4) G. Morrow and R. Russell: Am. Ceram. Soc. Bull., 64 (1985), No. 7 , 1007.

5) J. Hlinka, A. Cramb and D. Bright: 68th Steelmaking Conf. Proc., ISS, Warrendale, PA, (1985), 35.

6) C. Tomazin, E. Upton and R. Wallis: Iron Steelmaker, 13 (1986), 28

7) A. Gaston, R. Laura and M. Medina: Ironmaking Steelmaking, 18 (1991), 370.

8) F. Shklyar, V. Malkin, V. Korshunov and V. Sovetkin: Steel USSR, 21 (1991), 68.

9) A. Zoryk and P. Reid: Iron Steelmaker, 20 (1993), 21.

10) B. Olika and B. Bjorkman: Scand. J. Metall., 22 (1993), 213.

11) B. Barber, G. Watson and L. Bowden: Ironmaking Steelmaking, 21 (1994), 150.

12) P. Austin, S. O'Rourke, Q. He and A. Rex: 75th Steelmaking Conf. Proc., ISS, Warrendale, PA, (1992), 317.

13) T. Fredman and H. Saxen: Metall. Mater. Trans. B, 29B (1998), 651.

14) T. Fredman, J. Torrkulla and H. Saxen: Metall. Mater. Trans. B, 30B (1999), 323.

15) B. Olika, Y. Pan, B. Bjorkman and C. Grip: Scand. J. Metall., 25 (1996), 18.

16) J. Ludley and J. Szekely: J. Iron Steel Inst., 204 (1966), 12.

17) J. Szekely and R. Lee: Trans. Metall. Soc. AIME, 242 (1968), 961.

18) J. Szekely and J. Chen: Metall. Trans., 2 (1971), 1189.

19) O. Ilegbusi and J. Szekely: Trans. Iron Steel Inst. Jpn., 27 (1987), 563.

20) P. Austin, J. Camplin, J. Herbertson and I. Taggart: ISIJ Int., 32 (1992), 196.

21) Y. Pan, C. Grip and B. Bjorkman: Scand. J. Metall., 32 (2003), No. 2,71 .

22) H. Tian, Z. Mao and A. Wang: ISIJ Int., 49 (2009), No. 1, 58

23) N. Gupta and S. Chandra: ISIJ Int., 44 (2004), No. 9, 1517.

24) T. Okura, M. Kano, H. Kitada and N. Murata: CAMP-ISIJ, 24 (2011), 29.

25) A. Doucet, N. Freitas and N. Gordon: Sequential Monte Carlo Mehods in Practice, Springer-Verlag, New York, (2001), 1.

26) R. Kalman: J. Basic Eng., 82D (1960), 35.

27) C. Bishop: Pattern Recognition and Machine Learning, SpringerVerlag, New York, (2006), 359.

28) J. Spall: Introduction to Stochastic Search and Optimization: Estimation, Simulation and Control, Wiley, NJ, (2003), 176.

29) C. Bishop: Neural Networks for Pattern Recognition, Oxford University Press, UK, (1996), 116.

30) J. Friedman and W. Stuetzle: J. Am. Stat. Assoc., 76 (1981), 817.

31) L. Breiman, J. Friedman, R. Olshen and C. Stone: Classification and Regression Trees, Wadsworth, Belmont, (1984), 216.

32) L. Breiman: Mach. Learn., 45 (2001), 5. 